\title{
ORIGINAL ARTICLE Genetic and clinical factors predict lithium's effects on PER2 gene expression rhythms in cells from bipolar disorder patients
}

\author{
MJ McCarthy ${ }^{1,2,3}, \mathrm{H} \mathrm{Wei}^{1,2,3}, \mathrm{Z} \mathrm{Marnoy}^{1,2,3}, \mathrm{RM}$ Darvish $^{2,3}, \mathrm{DL} \mathrm{McPhie}^{4}$, BM Cohen $^{4}$ and DK Welsh ${ }^{1,2,3}$
}

Bipolar disorder (BD) is associated with abnormal circadian rhythms. In treatment responsive BD patients, lithium (Li) stabilizes mood and reduces suicide risk. Li also affects circadian rhythms and expression of 'clock genes' that control them. However, the extent to which BD, Li and the circadian clock share common biological mechanisms is unknown, and there have been few direct measurements of clock gene function in samples from BD patients. Hence, the role of clock genes in BD and Li treatment remains unclear. Skin fibroblasts from BD patients $(N=19)$ or healthy controls $(N=19)$ were transduced with Per2::/uc, a rhythmically expressed, bioluminescent circadian clock reporter gene, and rhythms were measured for 5 consecutive days. Rhythm amplitude and period were compared between BD cases and controls with and without Li. Baseline period was longer in BD cases than in controls. Li $1 \mathrm{~mm}$ increased amplitude in controls by 36\%, but failed to do so in BD cases. Li 10 mm lengthened period in both BD cases and controls. Analysis of clock gene variants revealed that PER3 and RORA genotype predicted period lengthening by Li, whereas GSK3 $\beta$ genotype predicted rhythm effects of Li, specifically among BD cases. Analysis of BD cases by clinical history revealed that cells from past suicide attempters were more likely to show period lengthening with Li 1 mm. Finally, Li enhanced the resynchronization of damped rhythms, suggesting a mechanism by which Li could act therapeutically in BD. Our work suggests that the circadian clock's response to Li may be relevant to molecular pathology of BD.

Translational Psychiatry (2013) 3, e318; doi:10.1038/tp.2013.90; published online 22 October 2013

Keywords: bipolar disorder; circadian rhythm; gene expression; lithium

\section{INTRODUCTION}

Bipolar disorder (BD) is a psychiatric illness characterized by disabling episodes of depression and mania that greatly increase the risk of suicide. ${ }^{1}$ The biological origins of $\mathrm{BD}$ are poorly characterized, but heritability is estimated to be 0.85 , suggesting a genetic etiology. ${ }^{2}$ Disturbances in sleep and activity are common in BD, leading to the hypothesis that circadian rhythm disruption is an essential feature of the illness (as reviewed in McCarthy and Welsh ${ }^{3}$ ). The cellular clock comprises $\sim 20$ 'clock genes' that form transcriptional-translational feedback loops to maintain $\sim 24 \mathrm{~h}$ cycles of gene expression and downstream processes. ${ }^{4}$ Variants in clock genes have been associated with $\mathrm{BD}$, major depression and seasonal affective disorder. ${ }^{3}$ Functional features of the molecular clock are defined by the transcriptional activity of CLOCK/BMAL1 heterodimers that stimulate gene expression through E-box elements in the promoters of $P E R 1 / 2 / 3$ and $C R Y 1 / 2$ genes, with subsequent feedback inhibition by CRY and PER proteins. In the forebrain, NPAS2 may substitute for CLOCK. $^{5,6}$ Secondary loops (e.g. RORA/B/C, REV-ERB $\alpha$ ), and posttranslational modifications by casein kinases $1 \delta / \varepsilon$ (encoded from CSNK1D/E) and glycogen synthase kinase $3 \beta$ (GSK3 $\beta$ ) regulate core loop functions, through alterations in protein stability and nuclear compartmentalization. ${ }^{7}$ Accordingly, inhibition of GSK3 $\beta$ increases amplitude and shortens period of gene expression rhythms. ${ }^{8-10}$

The mood stabilizer lithium (Li) often improves depression and mania, reduces suicide risk and normalizes daily rhythms in $B D$, but $\mathrm{BD}$ is heterogeneous, with Li-responsive and non-responsive subtypes that may differ in important ways. Among its molecular targets, Li inhibits GSK3 $\beta{ }^{11}$ a feature of the drug that may account for the effects on both mood and the clock. ${ }^{12}$ Indeed, some studies have identified genetic variants in GSK3 $\beta{ }^{13,14}$ (but see references 15 and 16), or clock gene substrates of GSK3 $\beta$ (e.g. REV-ERB $\alpha$ ) that predict clinical response to $\mathrm{Li}^{17,18} \mathrm{Li}$ increases the amplitude of PER2 rhythms in cells and tissue slices, ${ }^{10,19,20}$ a feature of the drug that may bolster weak rhythms in some cells. Unlike selective GSK3 $\beta$ inhibitors, Li lengthens the period of behavioral rhythms, ${ }^{21-23}$ a paradox that could indicate the presence of additional Li targets within the clock network. However, no study has adequately examined the connection between $\mathrm{Li}$ and circadian clock function in cells from BD patients. Therefore, it is unknown if $\mathrm{Li}$ affects rhythms distinctly in BD. The finding that fibroblasts and neurons have cell autonomous molecular clocks operating through similar mechanisms ${ }^{24}$ suggests that this question is amenable to study in skin fibroblasts from BD patients. ${ }^{25}$

Our principal hypothesis was that clock gene rhythms and their response to Li would differ in cells from BD patients compared to controls. As secondary hypotheses, we investigated whether clock gene variants and clinical subtypes of $\mathrm{BD}$ were associated with differences in rhythms. Supporting our hypotheses, we report that cells from BD patients have longer circadian rhythm periods, and show significant differences in response to $\mathrm{Li}$.

\section{METHODS}

Per2::/uc reporter

The Per2::Iuc lentiviral reporter gene was provided by Andrew Liu (University of Memphis), and has been described previously. ${ }^{24}$ All experiments used $\sim 1 \times 10^{7}$ infectious units/plate. Transduction efficiency was estimated to be $\sim 80-100 \%$.

\footnotetext{
${ }^{1}$ Psychiatry Service, Veterans Affairs San Diego Healthcare System, University of California, San Diego, CA, USA; ${ }^{2}$ Department of Psychiatry, University of California, San Diego, CA, USA; ${ }^{3}$ Center for Chronobiology, University of California, San Diego, CA, USA and ${ }^{4}$ Harvard McLean Hospital, Belmont, MA, USA. Correspondence: Dr MJ McCarthy, Psychiatry Service, Veterans Affairs San Diego Healthcare System, University of California, 3350 La Jolla Village Drive, MC 116A, San Diego, 92161 CA, USA. 


\section{Drugs}

Li chloride was purchased from Sigma. Stock Li solutions were made from sterile water at a concentration of $1 \mathrm{M}$ and used to make the appropriate drug-containing cell culture media.

Human subjects and cell lines

Subjects 18-65 years old were recruited from McLean Hospital (cases) and the surrounding community (healthy controls). BD cases were typically identified while inpatients on a psychiatric unit. All subjects provided informed consent and were paid to participate. Subjects were evaluated with a structured interview (SCID-Mini for DSM-IV) to establish diagnosis. All cases had a primary diagnosis of BD type I. Clinical features including age of onset, psychiatric family history, dysphoric/euphoric mania, past suicide attempts (PSA), alcohol/substance use history were extracted from the diagnostic interview. Most of the BD patients were on multiple psychotropic medications (mean 3.3). Medications at the time of biopsy were recorded (Supplementary Table S1), but details regarding past medication trials and treatment response were not available. Subjects were excluded if they were medically ill or had a history of adverse events with skin biopsies. Age-matched controls were excluded for any psychiatric diagnosis, or use of psychotropic medication.

Fibroblast cell lines were established from skin biopsies from the buttock. ${ }^{26}$ De-identified cell lines were subsequently sent to a collaborating laboratory for culture and analysis. At the time of experiments, cell lines had been passaged 3-10 times. Case-control matching was maintained during cell culture procedures and experiments.

\section{Cell culture and luminometry}

For reporter gene assays, cells were grown to confluence in 100-mm plates in standard culture media [DMEM with $10 \%$ fetal bovine serum (FBS), glutamine and antibiotics (penicillin, streptomycin and amphotericin)]. After 2 days, cells were dissociated with trypsin, and $\sim 10 \%$ of each culture was transferred into one of six identical $35-\mathrm{mm}$ plates (duplicates of vehicle, $\mathrm{Li}$ $1 \mathrm{mM}$ and $\mathrm{Li} 10 \mathrm{~mm}$ ) and stabilized for $48 \mathrm{~h}$. Afterwards, transduction with the Per2::Iuc construct proceeded over $48 \mathrm{~h}$ as cells were grown to confluence $\left(\sim 1.2 \times 10^{6}\right.$ cells/plate). Immediately before luminometer assay, growth medium was replaced with HEPES-buffered, serum-free recording media containing $1 \mathrm{~mm}$ luciferin (Biosyth International, Staad, Switzerland) as described previously. ${ }^{27}$ This procedure was sufficient to synchronize Per2::Iuc rhythms without serum shock. For experiments with Li (1 or $10 \mathrm{~mm}$ ), drug was continuously present in the growth medium, starting $48 \mathrm{~h}$ before recording, and throughout the recording period for a total exposure of $\sim 7$ days.

Rhythm measurements were conducted in $35 \mathrm{~mm}$ culture plates as previously described ${ }^{28}$ using a 32 -well luminometer (Actimetrics, Wilmette, IL, USA). Photoemissions were measured from duplicate plates of each sample for $70 \mathrm{~s}$, every $10 \mathrm{~min}$ for $\sim 5$ days. Temperature was maintained at $35^{\circ} \mathrm{C}$.

\section{Gene expression}

For analyses of BMAL1, CLOCK and NPAS2 expression, duplicate cultures $(N=7$ control, $10 \mathrm{BD})$ were grown in six-well plates. Thirty-six hours prior to collection, the medium was changed to $1 \mathrm{mM} \mathrm{Li}$ or vehicle containing DMEM to synchronize rhythms. Medium was then aspirated, and the plates were frozen at $-80^{\circ} \mathrm{C}$. RNA was prepared using a Qiagen (Hilden, Germany) RNeasy kit, following the instructions provided by the manufacturer. Using a high-capacity reverse transcriptase kit, CDNA ( $750 \mathrm{ng}$ ) was synthesized following the manufacturer's protocol. Taqman RT-PCR was conducted using a Bio-Rad CFX384 thermocycler with primers (Applied Biosystems, Foster City, CA, USA) targeting BMAL1 (ARNTL), CLOCK, NPAS2 and GAPDH, a non-rhythmic control gene suitable for circadian rhythm studies. ${ }^{29}$ Gene expression was measured against GAPDH using the comparative $\mathrm{Ct}$ method. ${ }^{30}$ Data were normalized to the vehicletreated control condition.

\section{DNA preparation and genotyping}

DNA was prepared from cells using a Qiagen DNeasy Kit following the manufacturer's protocol. One single-nucleotide polymorphism (SNP) from each of 14 clock genes was selected for study, based on minor allele frequencies that permit examination in small samples, and where possible, an association with a relevant mood phenotype (Table 1). Most genotyping was done using pre-designed Taqman assays (Applied Biosystems). For the
Table 1. Clock gene SNPs and MAF in BD cases and controls

\begin{tabular}{|c|c|c|c|c|}
\hline Gene & $S N P$ & $\begin{array}{l}\text { MAF } \\
\text { Cont }\end{array}$ & $\begin{array}{c}M A F \\
B D\end{array}$ & Notes \\
\hline BMAL1 & rs2279287 & 31.6 & 13.2 & $\begin{array}{l}\text { Assoc BD, AKA } \\
\text { ARNTL }^{38}\end{array}$ \\
\hline CLOCK & rs1801260 & 31.6 & 26.3 & $\begin{array}{l}\text { Assoc BD and } \\
\text { insomnia }\end{array}$ \\
\hline CRY1 & rs8192440 & 26.3 & 28.9 & Assoc Li response ${ }^{18}$ \\
\hline CRY2 & rs2037311 & 2.6 & 2.6 & \\
\hline CSNK1E & rs1534891 & 13.2 & 10.5 & \\
\hline GSK3 $\beta$ & rs334558 & 28.9 & 28.9 & Assoc Li response $\mathrm{e}^{13,14}$ \\
\hline NPAS2 & rs11541353 & 18.4 & 23.7 & $\begin{array}{l}\text { Assoc SAD, AKA } \\
\text { S471L }^{33}\end{array}$ \\
\hline $\begin{array}{l}\text { Rev- } \\
\text { Erb } \alpha\end{array}$ & rs2071427 & 18.4 & 18.4 & $\begin{array}{l}\text { Assoc Li response, } \\
\text { AKA NR1D1 }\end{array}$ \\
\hline PER1 & rs3027178 & 15.8 & 26.3 & \\
\hline PER2 & rs56013859 & 15.8 & 26.3 & $\begin{array}{l}\text { Assoc SAD, }{ }^{33} \text { AKA } \\
10870\end{array}$ \\
\hline PER3 & rs228729 & 36.8 & 26.3 & Assoc $\mathrm{BD}^{38}$ tags VNTR \\
\hline RORA & rs12912233 & 44.7 & 39.5 & Assoc depression ${ }^{31}$ \\
\hline
\end{tabular}

Abbreviations: Assoc, associated; AKA, also known as; MAF, minor allele frequency; SAD, seasonal affective disorder; SNP, single-nucleotide polymorphism; VNTR, variable number tandem repeat. In some instances, past illness associations and reference are indicated. Also known as (AKA) indicates alternate name for SNP or gene.

PER2 SNP rs56013859, a custom Taqman assay was purchased using published sequence. ${ }^{35} \mathrm{PCR}$ reactions were conducted as described above.

\section{Data analysis}

For each cell line, luminometry data were averaged and fitted to a damped sine curve by the least squares method using commercial software (Lumicycle Analysis, Actimetrics). To limit variability, the first 0.6 days were excluded from analysis. Data were filtered using moving average background subtraction. Rhythm parameters were defined as period, amplitude and goodness of fit as an index of rhythm robustness. Phase was correlated to period and for this reason, was not considered separately. For two-way analyses of rhythms, parameters were analyzed separately using an unpaired, two-tailed t-test or Mann-Whitney U-test. For analyses of Li-induced changes in rhythms, a two-way analysis of variance with repeated measures was conducted for each parameter. Correlations were established using Pearson's test. All analyses were conducted using GraphPad Prism version 5.0 (San Diego, CA, USA). P-values $<0.05$ were employed as the threshold for significance in primary analyses. The Benjamini-Hochberg correction for multiple comparisons employing a false discovery rate of $0.05^{36}$ was applied to secondary analyses using publicly available software (Q-Value). After this correction, $P$-values $<0.001$ remained significant. $P$-values $<0.05$ that fell short of significance after false discovery rate correction were reported as nominally significant.

\section{RESULTS}

\section{Baseline rhythm parameters}

Robust oscillations in Per2::/uc gene expression were present in all cell lines (goodness of fit $>80 \%$ ). Consistent with previous reports of peripheral cell cultures, rhythms were detectable in all cultures for $>5$ days before eventual rhythm damping, likely reflecting desynchronization. ${ }^{37}$ In our primary analysis examining rhythm parameters among BD cases and controls, rhythm amplitude was similar between groups. However, period was longer by $\sim 25 \mathrm{~min}$ in BD cells compared with controls (controls $25.1 \pm 0.2 \mathrm{~h}$ vs BD $25.5 \pm 0.2 \mathrm{~h}$, mean \pm s.e.m., $U=104.5, P<0.03$, Supplementary Table S2).

\section{Li treatment and rhythms}

$\mathrm{Li}$ is chronically dosed in BD patients to serum concentrations of $0.5-1.0 \mathrm{~mm}$, whereas past in vitro studies have used Li concentrations 

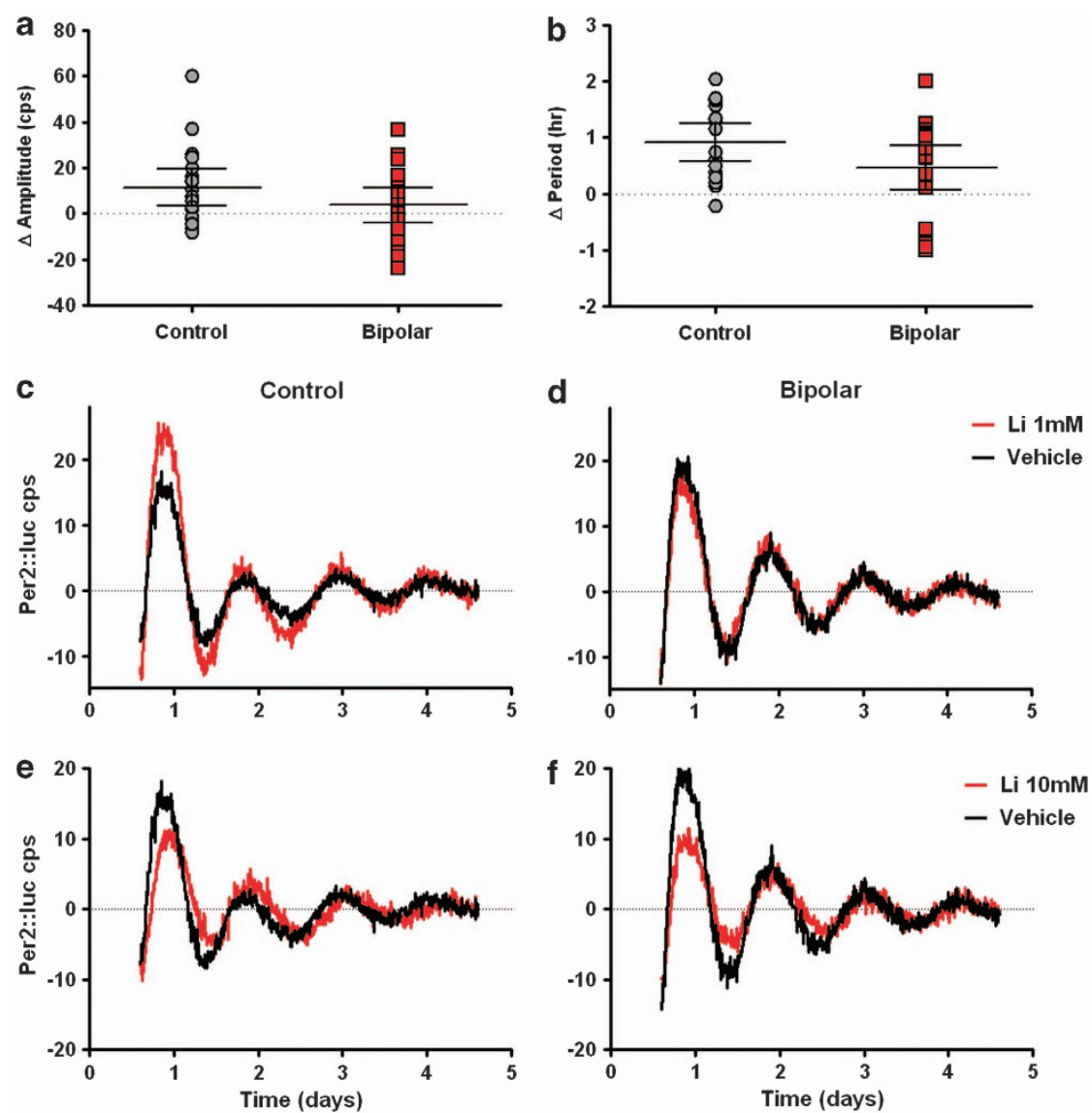

Figure 1. Fibroblasts from BD patients have an attenuated response to Li. Vehicle, Li $1 \mathrm{~mm}$ or Li $10 \mathrm{~mm}$ was included in the growth medium for $48 \mathrm{~h}$ prior to, and continuously during 5 days of recording. Rhythmic Per2::Iuc expression was measured as counts per second (cps) with baseline subtraction. (a) Amplitude increased in cells from control $(N=19, P<0.01)$, but not BD $(N=19, P=0.32)$ subjects after Li 1 mM (error bars indicate $95 \%$ confidence intervals). (b) There was a non-significant trend toward greater period lengthening in controls compared with BD cells after Li $10 \mathrm{~mm}$ (error bars indicate 95\% confidence intervals). Representative vehicle-treated (black) and Li-treated (red) rhythm traces are shown for representative control and BD cell lines at $1 \mathrm{~mm}(\mathbf{c}, \mathbf{d})$ and $10 \mathrm{~mm}(\mathbf{e}, \mathbf{f})$.

$>10 \mathrm{~mm}^{7,10}$ In order to investigate both conditions, we used Li concentrations of $1 \mathrm{~mm}$ and $10 \mathrm{~mm}$. While there was heterogeneity among individual samples, Li $1 \mathrm{~mm}$ on average did not lengthen period in either control or BD cells. Li $1 \mathrm{~mm}$ increased amplitude by $\sim 36 \%$ in control cells (31.7 vehicle vs $43.2 \mathrm{Li}, P<0.01$, Figure $1 \mathrm{a}$ ), but failed to significantly increase amplitude in BD cells (31.7 vehicle vs $35.5 \mathrm{Li}, P=0.38$, Supplementary Table S3, Figure $1 \mathrm{~b}$ ).

In agreement with previous studies, Li $10 \mathrm{~mm}$ caused period lengthening in control and BD cells (Supplementary Table S4, Figures $1 \mathrm{c}$ and $\mathrm{d}$ ), with a trend toward greater period lengthening in controls compared to $\mathrm{BD}(0.9 \mathrm{~h}$ control vs $0.4 \mathrm{~h} \mathrm{BD}, P=0.09)$. In contrast to the lower concentration, Li $10 \mathrm{~mm}$ increased the mean Per2::/uc signal, but significantly suppressed amplitude $(P<0.0001)$ after subtracting the non-rhythmic component. This amplitude reduction was similar between groups, with decreases of $27 \%$ and $37 \%$ for controls and BD respectively. There was no significant drug $\times$ genotype interaction.

\section{Analysis of CLOCK, BMAL1 and NPAS2 expression}

Rhythmic expression of PER2 is driven by heterodimeric CLOCK:BMAL1 or CLOCK:NPAS2 complexes binding to E-box promoter elements. Like PER2, BMAL1 and NPAS2 expression is rhythmic, but with a different phase, peaking $\sim 12 \mathrm{~h}$ earlier than PER2. While CLOCK has an important role in mediating PER2 rhythms, its expression can be rhythmic or constitutive depending on the tissue, and rhythmic expression is not essential for CLOCK's function. ${ }^{34}$ In an initial examination of the molecular mechanisms by which amplitude was increased after treatment with $\mathrm{Li} 1 \mathrm{~mm}$ in control but not BD cells, we measured expression of BMAL1, CLOCK and NPAS2 in fibroblasts $36 \mathrm{~h}$ after a synchronizing medium change, with or without Li $1 \mathrm{~mm}$ added to the medium. Based on the results of Per2::/uc experiments, this is a time when peak expression of all three genes is expected. However, at this time point there were no differences in BMAL1, CLOCK or NPAS2 expression between $\mathrm{BD}$ cases and controls either at baseline or after Li treatment (Supplementary Table S5). Because the heterodimer is the functional unit for the relevant gene products, and CLOCK and NPAS2 compete for BMAL1 binding, the ratios of CLOCK/BMAL1, CLOCK/NPAS2 and NPAS2/BMAL1 were each calculated (Supplementary Table S6). Li significantly decreased the ratio of CLOCK/NPAS2 $(P=0.02)$, and increased the ratio of NPAS2/ BMAL1 $(P=0.01)$, but these effects were similar among BD cases and controls. Therefore, although Li may increase the relative contribution of NPAS2 to PER2 regulation, we found no changes in peak BMAL1, CLOCK, or NPAS2 expression that might explain why Per2::IUc rhythm amplitude was increased by $\mathrm{Li} 1 \mathrm{mM}$ in control, but not BD cells.

Genetic variation in the clock and $\mathrm{Li}$

As a secondary analysis, we examined clock gene variants as predictors of Li-induced rhythm changes. Minor allele frequency and overall burden of variant clock gene alleles did not 

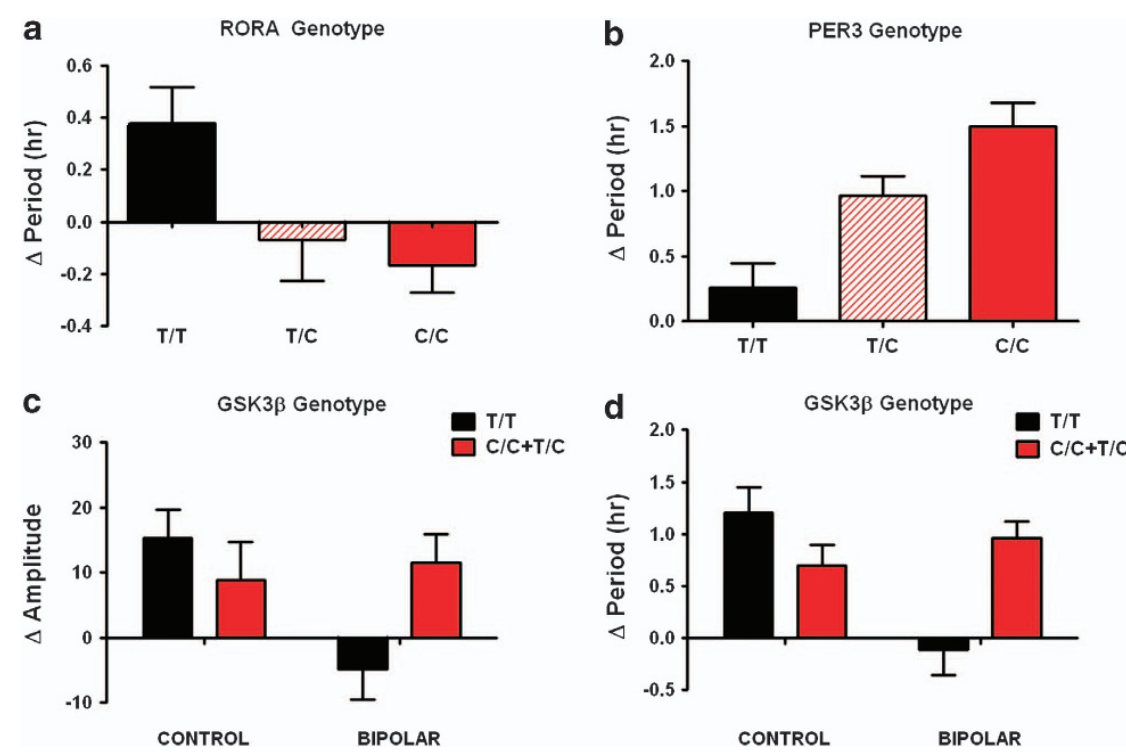

Figure 2. Rhythm modulation by $\mathrm{Li}$ is associated with clock gene variants. (a) Period lengthening after Li $1 \mathrm{~mm}$ for RORA rs 12912233 homozygous common (T/T), heterozygous $(\mathrm{C} / \mathrm{T})$ and homozygous variant $(\mathrm{C} / \mathrm{C})$ genotypes $[N=5 / 11 / 3$ (controls), $N=7 / 9 / 3(\mathrm{BD}), P<0.05]$. (b) Period lengthening after $\mathrm{Li} 10 \mathrm{~mm}$ for PER3 rs228729 homozygous common (T/T), heterozygous (T/C) and homozygous variant (C/C) genotypes [ $N=6 / 12 / 1$ (controls), $N=11 / 6 / 2(B D), P<0.001]$. In both cases $(\mathbf{a}, \mathbf{b})$, genotype effects were significant regardless of diagnosis so $\mathrm{BD}$ cases and controls have been combined. (c) Amplitude increase after $\mathrm{Li} 1 \mathrm{~mm}$ among GSK3 $\beta$ - 50T/C homozygous common (T/T) and rare variant carriers $(\mathrm{T} / \mathrm{C}$ and $\mathrm{C} / \mathrm{C}$ ). Two-way ANOVA revealed a significant genotype $\times$ diagnosis interaction $(P<0.05)$, but no significant difference between genotypes or diagnoses alone. (d) Period lengthening after Li $10 \mathrm{~mm}$. Two-way ANOVA revealed effect of $\mathrm{BD}(P<0.05)$, and significant genotype $\times$ diagnosis interaction $(P<0.001)$. For homozygous common/heterozygous/homozygous variant genotypes, $N=8 / 11 / 0($ controls $)$ and $N=9 / 9 / 1$ (BD), respectively. Error bars indicate s.e.m.

significantly differ between BD cases and controls (Table 1). However, when period and amplitude were examined as quantitative traits under baseline and Li-treated conditions, two of these variants were nominally associated with period lengthening, independent of BD diagnosis (RORA rs12912233, and PER3 rs228729, Figures 2a and b). Homozygotes for the common T allele in RORA showed period lengthening with $\mathrm{Li} 1 \mathrm{~mm}$, but heterozygous or homozygous carriers of the variant $C$ allele did not $(P<0.05)$. Carriers of the $P E R 3$ variant $C$ allele were more sensitive to the effect of $\mathrm{Li} 10 \mathrm{~mm}$ in an allelic copy number-dependent manner $(P<0.001)$. Interestingly, the RORA SNP has previously been associated with depression traits by genome-wide association study, ${ }^{31}$ and the PER3 variant has been associated with $\mathrm{BD} .^{38}$

GSK3 $\beta$ SNP rs334558 ( $-50 T / C)$ has previously been associated with clinical response to $\mathrm{Li}$ in both $\mathrm{BD}$ and major depression. ${ }^{13,14}$ With respect to rhythms, there were nominally significant interactions $(P<0.05)$ between GSK3 $\beta$ genotype and diagnosis: cells from $B D$ subjects carrying the variant $C$ allele showed increased amplitude in the presence of $\mathrm{Li} 1 \mathrm{~mm}$, similar to the controls of either genotype, but BD cells homozygous for the common $T$ allele did not (Figure 2c). GSK3 $\beta$ genotype also predicted period lengthening by Li $10 \mathrm{~mm}$, but again only in BD cell lines, with a main effect of diagnosis $(P<0.05)$, and a significant diagnosis $\times$ genotype interaction $(P<0.001)$. Control cells demonstrated period lengthening regardless of GSK3 $\beta$ genotype, BD cells with the homozygous T/T genotype showed no change in period, and cells with C/C or T/C GSK3 $\beta$ genotypes showed period lengthening, similar to controls (Figure 2d). Period and amplitude were not correlated in the study population as a whole, suggesting that this pattern of genetic association reflects non-redundant contributions of GSK3 $\beta$ to amplitude and period effects.

Clinical subtype associations

Clinical features have been used to predict Li response and suicide risk in $\mathrm{BD} .{ }^{39}$ We conducted secondary analyses of the rhythm data to assess differences among BD sub-phenotypes. Neither PSA, comorbid alcohol use disorder/substance use disorders, nor family history of BD in first degree relatives were associated with any difference in baseline rhythm parameters. Subjects with only euphoric manias (compared with sometimes/always dysphoric) had slightly less rhythm robustness (goodness of fit: $85 \%$ dysphoric, $78 \%$ euphoric, $P<0.05$ ), but did not differ in period or amplitude (Supplementary Table S7). Female sex was nominally associated with exclusively euphoric mania, and negatively associated with rhythm robustness (goodness of fit: female $75.7 \%$ vs male $84.8 \%$, $P<0.01$ ). Age, age of BD onset and illness duration did not correlate with any rhythm parameter. Among controls, age and sex did not correlate with any rhythm parameter.

Using the phenotypic and demographic variables above, we also examined the clock gene response to $\mathrm{Li}$ in $\mathrm{BD}$ cells by clinical features. Unlike controls and BD subjects considered collectively, BD subjects with a history of PSA demonstrated nominally significant period lengthening after treatment with $\mathrm{Li} 1 \mathrm{~mm}$ (Figure 3a). Under the same conditions, there was a trend towards amplitude increases in the PSA positive group, but it was not statistically significant (Figure $3 b$ ). However, when suicide history was assessed as a quantitative measure (number of PSAs), there were nominally significant correlations $(P<0.05)$ between PSA and period lengthening by Li $1 \mathrm{~mm}(r=0.47, P<0.05$, Figure $3 \mathrm{c})$. The correlation between PSA and amplitude increase by Li $1 \mathrm{~mm}$ was also significant $(r=0.50, P<0.05$, Figure $3 \mathrm{~d}$ ), but was strongly affected by a single data point with 4 PSAs, and was no longer significant when this subject was excluded. Comorbid alcohol use disorder/substance use disorder, family history or demographic variables were not associated with any changes in rhythm parameters after treatment with Li $1 \mathrm{~mm}$. With Li $10 \mathrm{~mm}$, amplitude was nominally higher in the dysphoric mania BD subgroup compared with those with euphoric mania only (23.4 vs 15.2 relative units, $P<0.05$ ). There were no further $B D$ subgroup differences in response to $\mathrm{Li} 10 \mathrm{~mm}$ when considering alcohol use disorder/substance use disorder, PSA, family history or age/sex. 

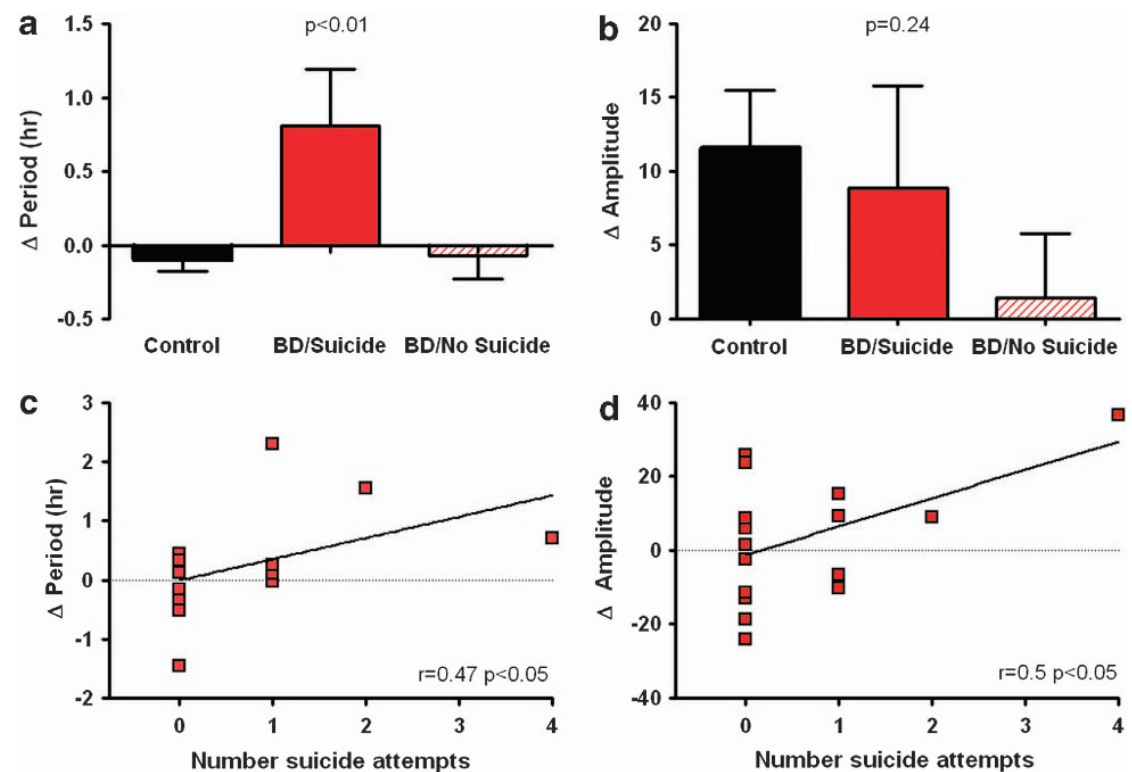

Figure 3. Suicide in $\mathrm{BD}$ is associated with period lengthening and amplitude increase after $\mathrm{Li} 1 \mathrm{~mm}$. When measured categorically, BD patients with a history of suicide were more sensitive to (a) period lengthening, but not (b) amplitude increase after treatment with $\mathrm{Li} 1 \mathrm{~mm}$. $P$-values indicate the results of 1-way ANOVA with $N=6$ PSA positive/13 PSA negative/19 controls. Error bars indicate s.e.m. When measured quantitatively, suicide history is significantly correlated with both (c) period lengthening and (d) amplitude increase after Li $1 \mathrm{~mm}$.

\section{Li augments rhythm resynchronization}

The effect of Li on amplitude suggests that the drug may bolster weak or failing rhythms in vulnerable cell populations, by improving rhythm amplitude. We tested this hypothesis in fibroblasts from control and BD subjects. Rhythms in untreated cells were allowed to damp over 5 days, and were then subjected to medium change, a stimulus commonly used to resynchronize rhythms in cell cultures. The medium change partially restored rhythms in the absence of $\mathrm{Li}$, but the resulting rhythm was typically weak with low amplitude and period exceeding the duration typically considered circadian $(>30 \mathrm{~h})$, consistent with poor synchronization among cells. When Li $10 \mathrm{~mm}$ was included in the medium, rhythms were more robustly restored, more closely resembling the initial signal, with higher amplitude and period closer to $24 \mathrm{~h}$ (Figures $4 \mathrm{a}-\mathrm{c}$ ). Statistical analysis revealed significant medium change $\times$ drug interactions for both amplitude $(P<0.04$, Figure 4a) and period ( $P<0.0001$, Figure $4 b)$, favoring rhythm restoration in Li-treated cells. The augmentation of rhythms by $\mathrm{Li}$ was similar in BD cases and controls ( $N=5 \mathrm{Li}$ treated of each), suggesting that despite the relative insensitivity of BD cells to $\mathrm{Li}$ compared with controls when rhythms are robust, BD cells remain Li sensitive when synchronization and/or amplitudes are weak and that therapeutic benefits of Li related to clock function in BD may be preferentially engaged when rhythms are attenuated.

\section{DISCUSSION}

\section{Overview}

Evidence from a range of sources supports the notion that the biological clock is perturbed in $\mathrm{BD}$, and that Li restores rhythms. ${ }^{3}$ However, definitive demonstration of cellular clock abnormalities in $\mathrm{BD}$ has proven elusive. We have shown in fibroblasts from BD patients that the cellular clock is functional, but generally runs slow, with a longer period, and is less sensitive to Li modulation. Among $\mathrm{BD}$ cases, variability in the rhythm response to $\mathrm{Li}$ is associated with clinical and genetic factors already known to be associated with clinical outcomes among BD patients. Although we do not know the clinical Li response history of our BD subjects, and cannot determine causality from these observations, the association of rhythm parameters with GSK3 $\beta$, a known genetic predictor of response, suggests that some of Li's therapeutic mechanisms may overlap with elements of the cellular clock, and could form the basis of a future biomarker for $\mathrm{BD}$ and/or $\mathrm{Li}$ response.

Effect of Li on rhythms

The period lengthening effect of $\mathrm{Li}$ on circadian rhythms is well established, ${ }^{21-23}$ and recent studies using bioluminescent cellular reporters have shown that $\mathrm{Li}$ increases amplitude. ${ }^{10,19,20}$ Our results generally agree with past reports, and extend these findings to include clinical samples from BD patients. We have also shown that the relative contribution of CLOCK and NPAS2 in governing rhythms may be affected by $\mathrm{Li}$, but occurs at concentrations below those required to alter period, and is unrelated to the differential modulation of amplitude observed in $\mathrm{BD}$ cases. Therefore, this level of transcriptional regulation by $\mathrm{Li}$ may be unimportant to PER2 regulation, but could conceivably influence the expression of other rhythmic genes (that is PER1/3, CRY1/2). However, our work also underscores the heterogeneity among individual responses, and differs in some respects from past work. Specifically, our results show that at higher concentrations of $\mathrm{Li}$, the effect on amplitude is complex with greater overall expression of Per2::Iuc, but suppression of the rhythmic component after background subtraction. Our observations in human and mouse cells indicate these differences are explained to some extent by chronic vs acute Li dosing, and species. Of particular importance to $\mathrm{BD}$, there may be important differences between neurons and fibroblasts, with indications that fibroblasts require higher concentrations of $\mathrm{Li}$ to influence rhythms. ${ }^{10}$

Rhythm differences between BD cases and controls

We found longer circadian periods of Per2::/uc rhythms in BD compared with controls. Long free running periods cause a delay in activity onset. Hence, this finding could point to a genetic basis for the nocturnal preference reported in BD patients who may experience the drive for peak activity later in the day. ${ }^{40} \mathrm{~A}$ previous study of BD fibroblasts using PCR-based measurements did not 


\section{6}
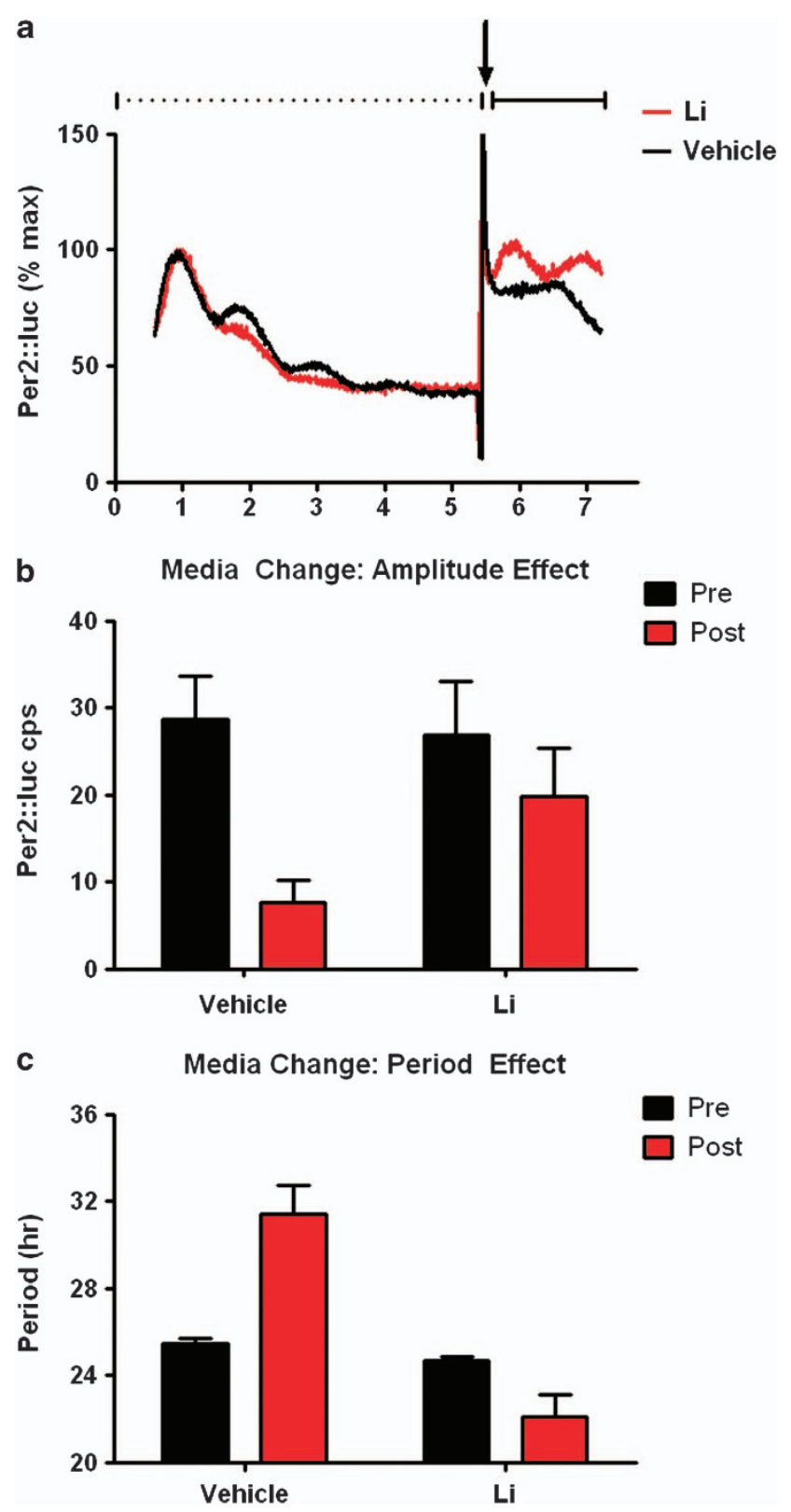

Figure 4. Li improves rhythm resynchronization. Cellular rhythms $(N=10$ each $\mathrm{BD}$ and control) were measured without treatment until damped (dashed line, day 1-5 in panel a). In order to restore rhythms, medium change with Li $10 \mathrm{~mm}$ (red) or vehicle (black) was performed on day 5.5 (arrow in panel a). Omitting the signal artifact associated with media change, rhythms were then measured another 2 days (solid line in panel a). After media change, Li-treated cells had (b) significantly higher rhythm amplitudes $(P<0.05)$, and (c) lower periods $(P<0.001)$ compared with vehicle-treated cells. Following media change, period in Li-treated cells was in the circadian range $(23.1 \pm 0.5 \mathrm{~h}$ (Ave \pm s.e.m.)); whereas period in the vehicle-treated cells was not (31.1 \pm 1.9 h (Ave \pm s.e.m.)).

find differences in circadian period, ${ }^{41}$ but the Per2::/uc method we used measures rhythms at a higher sampling density, and therefore the discordant results may be due to the increased sensitivity of our technique compared with older methods. The same study did report reduced amplitudes of BMAL1, Rev-Erb $\alpha$ and DBP rhythms. ${ }^{41}$ Similarly, amplitude abnormalities in behavior have been reported in actigraphy studies of euthymic BD patients. ${ }^{42}$ We did not find evidence of lower Per2::/uc amplitude at baseline in BD, and did not measure amplitude of BMAL1, Rev-Erb $\alpha$ or DBP rhythms. However, we report that Li increases Per2::/uc amplitude at therapeutic concentrations, consistent with the idea that $\mathrm{Li}$ could reverse putative rhythm defects reported by others.

In general, cells from BD patients were less responsive to Li's modulation of amplitude (and perhaps period) than were controls. Taken together, these findings could indicate that the clock in BD is not particularly impaired at baseline, but may be less flexible, and not as responsive to conditions that would normally shift the clock. This finding might be relevant to observations that disruptions to light-dark or sleep schedules can trigger mood episodes in individuals susceptible to BD. ${ }^{43}$ Alternatively, past exposure to $\mathrm{Li}$ and/or other psychotropic medications may have had lasting effects on the Li sensitivity of cellular rhythms. As BD cell line donors were more likely to be exposed to medication than controls, this could, in principle, explain the differences between groups, but requires the presence of a long-lasting mechanism to explain the result after multiple passages of cell culture. To date, no such mechanism has been described. Regardless of the mechanism, when rhythms were attenuated by damping, both $\mathrm{BD}$ and control cells showed a similar degree of improvement in rhythms after Li. Interestingly, valproic acid has been reported to increase rhythm amplitude. ${ }^{19}$ Although this drug has not been examined in BD cell lines, the similar amplitudeenhancing effects of $\mathrm{Li}$ and valproic acid suggest the possibility of a common circadian mechanism among mood stabilizers. Further, despite the insensitivity of the BD clock to Li, these results suggest that low amplitude clocks may become desynchronized, and benefit from amplitude-enhancing treatments to facilitate resynchronization. This may be relevant to cell populations in the brain that govern mood, some of which express clock genes and/or have circadian oscillators (as reviewed in McCarthy and Welsh ${ }^{3}$ ). In contrast, the effect of Li on period may worsen the already longer period in BD, further delaying activity onset, and the period effect is prominent only at supratherapeutic concentrations. For these reasons, we hypothesize that any therapeutic effect of $\mathrm{Li}$ on the clock is primarily due to modulation of amplitude, and not period.

\section{Li-responsive BD and circadian rhythms}

$\mathrm{BD}$ is commonly divided into Li-responsive and non-responsive types. ${ }^{44}$ We do not have data on the clinical Li-responsiveness of the BD patients from which our cells were derived, but we did examine clinical and genetic features previously shown to be associated with Li-responsiveness. Classical $\mathrm{Li}$ responders have few comorbid psychiatric diagnoses, a positive family history of $\mathrm{BD}$, and a preponderance of euphoric manias. ${ }^{39}$ We did not find clear differences in rhythms across these clinical dimensions. However, genetic factors, including some that predict clinical $\mathrm{Li}$ response, were associated with Li-induced changes in rhythms. We have shown previously that expression of clock genes, and genes strongly regulated by the clock are preferentially modulated by $\mathrm{Li}^{45}$ Moreover, clinical $\mathrm{Li}$ response has been previously associated with genetic variants in the circadian clock, including the -50 T/C SNP in GSK3 $\beta$, and Rev-Erb $\alpha .{ }^{17,18}$ The $-50 T / C$ SNP is thought to affect the GSK3 $\beta$ promoter, with the $C$ allele associated with lower gene expression, perhaps phenocopying the effects of $\mathrm{Li}$ inhibition of the enzyme. ${ }^{18}$ In the present study, GSK3 $\beta$ genotype was associated with amplitude increase in response to $\mathrm{Li} 1 \mathrm{~mm}$, and also with period lengthening at Li $10 \mathrm{~mm}$, but only in BD cell lines. For both parameters, there was a genotype $\times$ diagnosis interaction, suggesting that the function of GSK3 $\beta$ in rhythm regulation differs in control and BD cells. This finding suggests that GSK3 $\beta$ may have a distinctive role in $\mathrm{BD}$, consistent with numerous reports implicating it as a pathogenic factor in the illness. ${ }^{46-50}$ 
Functional implications for $\mathrm{Li}$ and genetic variation in the clock In addition to the BD-specific differences associated with GSK3 $\beta$, we identified two variants in PER3 and RORA that were associated with period length after $\mathrm{Li}$ in both $\mathrm{BD}$ cases and controls, suggesting these may reflect variation in the clock that is not necessarily pathogenic for $\mathrm{BD}$, but may influence the course of Li treatment. The RORA variant is intronic, with presumptive regulatory function. Of interest, other variants in RORA have been independently associated with anti-depressant response, ${ }^{51}$ post-traumatic stress disorder ${ }^{52}$ and $\mathrm{BD}^{53}$ suggesting the RORA gene may be vulnerable in multiple psychiatric conditions. The PER3 variant is in almost perfect linkage disequilibrium with the variable number tandem repeat (VNTR) polymorphism. The VNTR is predicted to alter protein coding sequence of PER3, changing the number of sites available for casein kinase $1 \varepsilon$ phosphorylation. ${ }^{54}$ In addition to $\mathrm{BD}^{55}$ the VNTR has been associated with diurnal preference, and sleep abnormalities. ${ }^{56}$ Therefore, while the effect of this PER3 variant on period in our experiment is similar between BD cases and controls, it too may have effects on illness progression or treatment, especially in the context of therapeutic Li use.

\section{Suicide and circadian rhythms}

$\mathrm{Li}$ has been identified as an anti-suicide drug, reducing risk in BD by fivefold. ${ }^{57}$ There is evidence that suicidal behavior is genetically transmitted $^{58,59}$ and segregates independently of BD. ${ }^{60}$ Diurnal variation in suicide has been suggested, with reports of both nighttime ${ }^{61}$ and morning peaks in incidence. ${ }^{62}$ More consistently, suicides have been shown to have a seasonal pattern with peaks in spring and autumn. ${ }^{63}$ A recent study found PER1 among several downregulated genes in the prefrontal cortex of suicide victims. ${ }^{64}$ In this context, it is interesting that our BD subjects with a suicide history were especially sensitive to Li's effects, suggesting that the clock genes may be among those underlying suicide, the most severe pathological feature of BD.

\section{Study limitations}

Peripheral clocks recapitulate central features of cellular clocks in the brain, but tissue specific factors may be important. Furthermore, our study was small, and underpowered to examine clinical and genetic sub-phenotypes in detail. Due to the number of secondary analyses conducted; there is an increased risk of falsepositive associations, and replication will be required. Future efforts will be directed toward testing our novel hypotheses in the context of large, well-powered studies that are amenable to detailed analyses.

\section{CONFLICT OF INTEREST}

The authors declare no conflict of interest.

\section{ACKNOWLEDGMENTS}

The authors thank Dost Öngür for his assistance in subject recruitment, and Caroline Nievergelt for providing statistical advice. MJM is supported by a Veterans Affairs Career Development Award (1IK2BX001275). DKW receives support from a Veterans Affairs Merit Award (1101BX001146), NIMH (R01 MH082945) and NARSAD Young Investigator Award. BC is the recipient of a NARSAD Distinguished Investigator Award. The funders had no role in the analysis, decision to publish or preparation of the manuscript.

\section{REFERENCES}

1 Oquendo MA, Currier D, Liu SM, Hasin DS, Grant BF, Blanco C. Increased risk for suicidal behavior in comorbid bipolar disorder and alcohol use disorders: results from the National Epidemiologic Survey on Alcohol and Related Conditions (NESARC). J Clin Psychiatry 2010; 71: 902-909.
2 McGuffin P, Rijsdijk F, Andrew M, Sham P, Katz R, Cardno A. The heritability of bipolar affective disorder and the genetic relationship to unipolar depression. Arch Gen Psychiatry 2003; 60: 497-502.

3 McCarthy MJ, Welsh DK. Cellular circadian clocks in mood disorders. J Biol Rhythms 2012; 27: 339-352.

4 Takahashi JS, Hong HK, Ko CH, McDearmon EL. The genetics of mammalian circadian order and disorder: implications for physiology and disease. Nat Rev Genet 2008; 9: 764-775.

5 Reick M, Garcia JA, Dudley C, McKnight SL. NPAS2: an analog of clock operative in the mammalian forebrain. Science 2001; 293: 506-509.

6 DeBruyne JP, Weaver DR, Reppert SM. CLOCK and NPAS2 have overlapping roles in the suprachiasmatic circadian clock. Nat Neurosci 2007; 10: 543-545.

7 Yin L, Wang J, Klein PS, Lazar MA. Nuclear receptor Rev-erbalpha is a critical lithium-sensitive component of the circadian clock. Science 2006; 311: 1002-1005.

8 Reischl S, Kramer A. Kinases and phosphatases in the mammalian circadian clock. FEBS Lett 2011; 585: 1393-1399.

9 Hirota T, Lewis WG, Liu AC, Lee JW, Schultz PG, Kay SA. A chemical biology approach reveals period shortening of the mammalian circadian clock by specific inhibition of GSK-3beta. Proc Natl Acad Sci USA 2008; 105: 20746-20751.

10 Li J, Lu WQ, Beesley S, Loudon AS, Meng QJ. Lithium impacts on the amplitude and period of the molecular circadian clockwork. PLoS One 2012; 7: e33292.

11 Klein PS, Melton DA. A molecular mechanism for the effect of lithium on development. Proc Natl Acad Sci USA 1996; 93: 8455-8459.

12 litaka C, Miyazaki K, Akaike T, Ishida N. A role for glycogen synthase kinase-3beta in the mammalian circadian clock. J Biol Chem 2005; 280: 29397-29402.

13 Benedetti F, Serretti A, Pontiggia A, Bernasconi A, Lorenzi C, Colombo C et al. Long-term response to lithium salts in bipolar illness is influenced by the glycogen synthase kinase 3-beta -50 T/C SNP. Neurosci Lett 2005; 376: 51-55.

14 Adli M, Hollinde DL, Stamm T, Wiethoff K, Tsahuridu M, Kirchheiner J et al. Response to lithium augmentation in depression is associated with the glycogen synthase kinase 3-beta -50T/C single nucleotide polymorphism. Biol Psychiatry 2007; 62: 1295-1302.

15 Michelon L, Meira-Lima I, Cordeiro Q, Miguita K, Breen G, Collier D et al. Association study of the INPP1, 5HTT, BDNF, AP-2beta and GSK-3beta GENE variants and restrospectively scored response to lithium prophylaxis in bipolar disorder. Neurosci Lett 2006; 403: 288-293.

16 Szczepankiewicz A, Rybakowski JK, Suwalska A, Skibinska M, Leszczynska-Rodziewicz A, Dmitrzak-Weglarz $M$ et al. Association study of the glycogen synthase kinase-3beta gene polymorphism with prophylactic lithium response in bipolar patients. World J Biol Psychiatry 2006; 7: 158-161.

17 Campos-de-Sousa S, Guindalini C, Tondo L, Munro J, Osborne S, Floris G et al. Nuclear receptor rev-erb-\{alpha\} circadian gene variants and lithium carbonate prophylaxis in bipolar affective disorder. J Biol Rhythms 2010; 25: 132-137.

18 McCarthy MJ, Nievergelt CM, Shekhtman T, Kripke DF, Welsh DK, Kelsoe JR. Functional genetic variation in the Rev-Erbalpha pathway and lithium response in the treatment of bipolar disorder. Genes Brain Behav 2011; 10: 852-861.

19 Johansson AS, Brask J, Owe-Larsson B, Hetta J, Lundkvist GB. Valproic acid phase shifts the rhythmic expression of Period2::Luciferase. J Biol Rhythms 2011; 26: 541-551.

20 Osland TM, Ferno J, Havik B, Heuch I, Ruoff P, Laerum OD et al. Lithium differentially affects clock gene expression in serum-shocked NIH-3T3 cells. J Psychopharmacol 2011; 25: 924-933.

21 Kripke DF, Judd LL, Hubbard B, Janowsky DS, Huey LY. The effect of lithium carbonate on the circadian rhythm of sleep in normal human subjects. Biol Psychiatry 1979; 14: 545-548.

22 Kripke DF, Wyborney VG. Lithium slows rat circadian activity rhythms. Life Sci 1980; 26: 1319-1321.

23 Welsh DK, Moore-Ede MC. Lithium lengthens circadian period in a diurnal primate, Saimiri sciureus. Biol Psychiatry 1990; 28: 117-126.

24 Liu AC, Welsh DK, Ko CH, Tran HG, Zhang EE, Priest AA et al. Intercellular coupling confers robustness against mutations in the SCN circadian clock network. Cell 2007; 129: 605-616.

25 Brown SA, Fleury-Olela F, Nagoshi E, Hauser C, Juge C, Meier CA et al. The period length of fibroblast circadian gene expression varies widely among human individuals. PLoS Biol 2005; 3: e338.

26 Takashima A. Establishment of fibroblast cultures. Curr Protoc Cell Biol 2001, Chapter 2: Unit 2.1.1-2.1.12.

27 Yamazaki S, Takahashi JS. Real-time luminescence reporting of circadian gene expression in mammals. Methods Enzymol 2005; 393: 288-301.

28 Welsh DK, Imaizumi T, Kay SA. Real-time reporting of circadian-regulated gene expression by luciferase imaging in plants and mammalian cells. Methods Enzymol 2005; 393: 269-288.

29 Kosir R, Acimovic J, Golicnik M, Perse M, Majdic G, Fink M et al. Determination of reference genes for circadian studies in different tissues and mouse strains. $B M C$ Mol Biol 2010; 11: 60. 
30 Schmittgen TD, Livak KJ. Analyzing real-time PCR data by the comparative $C(T)$ method. Nat Protoc 2008; 3: 1101-1108.

31 Terracciano A, Tanaka T, Sutin AR, Sanna S, Deiana B, Lai S et al. Genome-wide association scan of trait depression. Biol Psychiatry 2010; 68: 811-817.

32 Benedetti F, Dallaspezia S, Fulgosi MC, Lorenzi C, Serretti A, Barbini B et al. Actimetric evidence that CLOCK 3111 T/C SNP influences sleep and activity patterns in patients affected by bipolar depression. Am J Med Genet B Neuropsychiatr Genet 2007; 144B: 631-635.

33 Partonen T, Treutlein J, Alpman A, Frank J, Johansson C, Depner M et al. Three circadian clock genes Per2, Arntl, and Npas2 contribute to winter depression. Ann Med 2007; 39: 229-238.

34 Wyse CA, Coogan AN. Impact of aging on diurnal expression patterns of CLOCK and BMAL1 in the mouse brain. Brain Res 2010; 1337: 21-31.

35 Kovanen L, Saarikoski ST, Haukka J, Pirkola S, Aromaa A, Lonnqvist J et al. Circadian clock gene polymorphisms in alcohol use disorders and alcohol consumption. Alcohol Alcohol 2010; 45: 303-311.

36 Storey JD. A direct approach to false discovery rates. J Royal Statistic Soc. Series B 2002; 64: 479-498.

37 Welsh DK, Yoo SH, Liu AC, Takahashi JS, Kay SA. Bioluminescence imaging of individual fibroblasts reveals persistent, independently phased circadian rhythms of clock gene expression. Curr Biol 2004; 14: 2289-2295.

38 Nievergelt CM, Kripke DF, Barrett TB, Burg E, Remick RA, Sadovnick AD et al. Suggestive evidence for association of the circadian genes PERIOD3 and ARNTL with bipolar disorder. Am J Med Genet B Neuropsychiatr Genet 2006; 141B: 234-241.

39 Grof P. Selecting effective long-term treatment for bipolar patients: monotherapy and combinations. J Clin Psychiatry 2003; 64(Suppl 5): 53-61.

40 Wood J, Birmaher B, Axelson D, Ehmann M, Kalas C, Monk K et al. Replicable differences in preferred circadian phase between bipolar disorder patients and control individuals. Psychiatry Res 2009; 166: 201-209.

41 Yang S, Van Dongen HP, Wang K, Berrettini W, Bucan M. Assessment of circadian function in fibroblasts of patients with bipolar disorder. Mol Psychiatry 2009; 14 143-155.

42 Jones SH, Hare DJ, Evershed K. Actigraphic assessment of circadian activity and sleep patterns in bipolar disorder. Bipolar Disord 2005; 7: 176-186.

43 Wehr TA. Sleep loss: a preventable cause of mania and other excited states. J Clin Psychiatry 1989; 50 Suppl: 8-16.

44 McCarthy MJ, Leckband SG, Kelsoe JR. Pharmacogenetics of lithium response in bipolar disorder. Pharmacogenomics 2010; 11: 1439-1465.

45 McCarthy MJ, Nievergelt CM, Kelsoe JR, Welsh DK. A survey of genomic studies supports association of circadian clock genes with bipolar disorder spectrum illnesses and lithium response. PLoS One 2012; 7: e32091.

46 Prickaerts J, Moechars D, Cryns K, Lenaerts I, van Craenendonck H, Goris I et al. Transgenic mice overexpressing glycogen synthase kinase 3beta: a putative model of hyperactivity and mania. J Neurosci 2006; 26: 9022-9029.

47 Gould TD, Einat H, O'Donnell KC, Picchini AM, Schloesser RJ, Manji HK. Betacatenin overexpression in the mouse brain phenocopies lithium-sensitive behaviors. Neuropsychopharmacology 2007; 32: 2173-2183.

48 Karege F, Perroud N, Burkhardt S, Schwald M, Ballmann E, La Harpe R et al. Alteration in kinase activity but not in protein levels of protein kinase $B$ and glycogen synthase kinase-3beta in ventral prefrontal cortex of depressed suicide victims. Biol Psychiatry 2007; 61: 240-245.
49 Lachman HM, Pedrosa E, Petruolo OA, Cockerham M, Papolos A, Novak T et al. Increase in GSK3beta gene copy number variation in bipolar disorder. Am J Med Genet B Neuropsychiatr Genet 2007; 144B: 259-265.

50 Benedetti F, Bollettini I, Barberi I, Radaelli D, Poletti S, Locatelli C et al. Lithium and GSK3-beta promoter gene variants influence white matter microstructure in bipolar disorder. Neuropsychopharmacology 2013; 38: 313-327.

51 Garriock HA, Kraft JB, Shyn SI, Peters EJ, Yokoyama JS, Jenkins GD et al. A genomewide association study of citalopram response in major depressive disorder. Biol Psychiatry 2010; 67: 133-138.

52 Logue MW, Baldwin C, Guffanti G, Melista E, Wolf EJ, Reardon AF et al. A genomewide association study of post-traumatic stress disorder identifies the retinoidrelated orphan receptor alpha (RORA) gene as a significant risk locus. Mol Psychiatry 2012; 18(8): 937-942.

53 Smith EN, Bloss CS, Badner JA, Barrett T, Belmonte PL, Berrettini W et al. Genomewide association study of bipolar disorder in European American and African American individuals. Mol Psychiatry 2009; 14: 755-763.

54 Archer SN, Robilliard DL, Skene DJ, Smits M, Williams A, Arendt J et al. A length polymorphism in the circadian clock gene Per3 is linked to delayed sleep phase syndrome and extreme diurnal preference. Sleep 2003; 26: 413-415.

55 Benedetti F, Dallaspezia S, Colombo C, Pirovano A, Marino E, Smeraldi E. A length polymorphism in the circadian clock gene Per3 influences age at onset of bipolar disorder. Neurosci Lett 2008; 445: 184-187.

56 Dijk DJ, Archer SN. PERIOD3, circadian phenotypes, and sleep homeostasis. Sleep Med Rev 2010; 14: 151-160.

57 Baldessarini RJ, Tondo L, Davis P, Pompili M, Goodwin FK, Hennen J. Decreased risk of suicides and attempts during long-term lithium treatment: a meta-analytic review. Bipolar Disord 2006; 8: 625-639.

58 Statham DJ, Heath AC, Madden PA, Bucholz KK, Bierut L, Dinwiddie SH et al. Suicidal behaviour: an epidemiological and genetic study. Psychol Med 1998; 28: 839-855.

59 Fu Q, Heath AC, Bucholz KK, Nelson EC, Glowinski AL, Goldberg J et al. A twin study of genetic and environmental influences on suicidality in men. Psychol Med 2002; 32: 11-24.

60 Schulze TG, Hedeker D, Zandi P, Rietschel M, McMahon FJ. What is familial about familial bipolar disorder? Resemblance among relatives across a broad spectrum of phenotypic characteristics. Arch Gen Psychiatry 2006; 63: 1368-1376.

61 Valtonen $\mathrm{H}$, Suominen K, Partonen T, Ostamo A, Lonnqvist J. Time patterns of attempted suicide. J Affect Disord 2006; 90: 201-207.

62 Preti A, Miotto P. Diurnal variations in suicide by age and gender in Italy. $J$ Affect Disord 2001; 65: 253-261.

63 Christodoulou C, Douzenis A, Papadopoulos FC, Papadopoulou A, Bouras G, Gournellis R et al. Suicide and seasonality. Acta Psychiatr Scand 2012; 125: 127-146.

64 Sequeira A, Morgan L, Walsh DM, Cartagena PM, Choudary P, Li J et al. Gene expression changes in the prefrontal cortex, anterior cingulate cortex and nucleus accumbens of mood disorders subjects that committed suicide. PLoS One 2012; 7 e35367.

c) (1) $\Theta$ This work is licensed under a Creative Commons Attributioncc) NonCommercial-NoDerivs 3.0 Unported License. To view a copy of this license, visit http://creativecommons.org/licenses/by-nc-nd/3.0/

Supplementary Information accompanies the paper on the Translational Psychiatry website (http://www.nature.com/tp) 